\title{
Hard-X-Ray Directional Dark-Field Imaging Using the Speckle Scanning Technique
}

\author{
Hongchang Wang, 'Yogesh Kashyap, and Kawal Sawhney \\ Diamond Light Source, Harwell Science and Innovation Campus, Didcot, Oxfordshire OX11 ODE, United Kingdom
}

(Received 14 October 2014; published 11 March 2015)

\begin{abstract}
$\mathrm{X}$-ray dark-field imaging can provide inaccessible and complementary information compared to conventional absorption contrast imaging. However, extraction of the dark-field signal is difficult, and sophisticated optics are often required. In this Letter, we report a novel approach to generate high-quality dark-field images using a simple membrane. The dark-field image is extracted from the maximum correlation coefficient by applying a cross-correlation algorithm to a stack of speckle images collected by scanning a membrane in a transverse direction to the incident $\mathrm{x}$-ray beam. The new method can also provide directional dark-field information, which is extremely useful for the study of strongly ordered systems. The potential of the proposed technique for nondestructive $\mathrm{x}$-ray imaging is demonstrated by imaging representative samples.
\end{abstract}

DOI: 10.1103/PhysRevLett.114.103901

PACS numbers: 42.30.Ms, 42.30.Va, 52.59.Px, 87.59.-e

X-ray absorption contrast imaging has been widely used in medical diagnostics and material science. Phase-contrast imaging can further enhance the sensitivity for samples with weak absorption. X-ray dark-field imaging, which is related to the subpixel structural anisotropy or variations in the electron density in samples on length scales smaller than the detector resolution, provides complementary mechanisms to reveal subtle features of the material under study [1]. Even though dark-field imaging is well known in visible light and electron microscopy, it has taken nearly a century after the discovery of $\mathrm{x}$ rays for it to be realized in the x-ray regime. Initially, it was demonstrated using analyzer crystals [2], but the dark-field signal was weak due to the low bandpass $\left(\sim 10^{-4}\right)$ of crystal optics. Over the last decade, dark-field imaging has increased in popularity after successful demonstrations using grating interferometry [3-5]. Directional dark-field imaging shows great potential for the application in mammography, emphysema diagnosis, and material study [6-8]. Recently, the edge-illumination technique was also successfully used to detect the dark-field signal with incoherent illumination $[9,10]$. However, most existing dark-field imaging techniques involve complicated optical elements, such as perfect crystals, precision gratings, or sophisticated masks. To overcome these limitations, the $\mathrm{x}$-ray near-field speckle technique was demonstrated to retrieve the dark-field image by scanning a simple phase object (a membrane or abrasive paper) [11]. Unfortunately, this approach is time consuming as two-dimensional (2D) raster scans involve acquiring a large number of images. Furthermore, it is not appropriate to treat the standard

Published by the American Physical Society under the terms of the Creative Commons Attribution 3.0 License. Further distribution of this work must maintain attribution to the author(s) and the published article's title, journal citation, and DOI. deviation as a dark-field signal (see the Supplemental Material [12]). Recently, an alternative method has been proposed to extract the dark-field image using only a few images [13]. Nevertheless, the spatial resolution of this method is poor, and the directional dark-field information is not accessible. In this Letter, we describe a new algorithm to extract the directional dark-field signal from the maximum correlation coefficient by applying normalized cross-correlation algorithms. We demonstrate that pixelwise analysis of dark-field images can be performed by scanning a phase object in only one dimension (1D).

When an object consisting of high-spatial-frequency features is placed into a coherent or partially coherent photon beam, a speckle pattern is generated by the combination of numerous waves of the same frequency but with different phases and amplitudes. In the x-ray near-field region $(z \leq 2 \pi \xi d / \lambda$, where $\lambda$ is the wavelength, $\xi$ is the coherence length, and $d$ is the average pore size of the membrane or scattering particle), the speckle size does not depend on the distance from the phase membrane or the $\mathrm{x}$-ray energy $[14,15]$. This remarkable property allows the near-field speckle to access $\mathrm{x}$-ray scattering information in the low- $q$ region, thereby enabling investigation of inhomogeneous systems [16]. The key idea behind using phase membranes is to act as a high-frequency wave front marker and enable the geometrical path of each ray to be uniquely tracked as it passes through the sample. When a phase membrane is scanned along the horizontal (vertical) direction $x_{p}\left(y_{p}\right)$, the intensity signal $I(l, m)$ in each pixel $(l, m)$ in the detector plane oscillates as a function of $x_{p}\left(y_{p}\right)$. The amplitude of the speckle pattern depends on the degree of coherence of the X-ray beam [17], which is degraded by small-angle scattering by the specimen, thereby broadening the speckle pattern. Variation in the intensity of the speckle pattern due to this broadening can, therefore, be used to infer the change in beam coherence induced by the specimen. 
The fundamental principle of the method presented here is to evaluate the maximum correlation coefficient distribution for each pixel with its neighboring pixel from a stack of speckle images captured with and without the specimen. To analyze such a coherence change quantitatively, the intensity oscillation for pixel $(l, m)$ in a Fourier series is

$$
\begin{aligned}
& I\left(l, m, x_{p}\right)=\sum_{n=0}^{N} a_{n}^{h}(l, m) \exp \left[b_{n}^{h}(l, m) x_{p}+\varphi_{n}^{h}(l, m)\right], \\
& I\left(l, m, y_{p}\right)=\sum_{n=0}^{N} a_{n}^{v}(l, m) \exp \left[b_{n}^{v}(l, m) y_{p}+\varphi_{n}^{v}(l, m)\right],
\end{aligned}
$$

where $h$ and $v$ denote horizontal and vertical scans, and $n$ is the order of the Fourier series. For simplicity, only the vertical scan case is discussed below, and we drop the subscripts $h$ and $v$ to aid clarity. $a_{n}, b_{n}$, and $\varphi_{n}$ are the appropriate Fourier coefficients. The values of all coefficients depend on the order number and the pixel position. As described in Ref. [11], grating interferometry can be considered as a special case only if Eq. (1) is approximated to first order. In this instance, the constant $b_{1}$ is related to the analyzer grating period, and the amplitude coefficients $a_{0}$, $a_{1}$, and phase shift $\varphi_{1}$ can be retrieved by performing $1 \mathrm{D}$ discrete fast Fourier transforms for each pixel [18]. Specklebased imaging can be understood as a general case where all orders are included to adequately describe the observed speckle intensity oscillations. For vertical scanning mode, the coefficients $b_{n}$ for neighboring pixels $(l, m)$ and $\left(l, m^{\prime}\right)$ are approximately equal, whereas the differences in coefficients $a_{n}$ can be quantified by the correlation coefficient $\gamma\left(l, m, y_{p}\right)$. This is typically performed at every step by subtracting the mean and dividing by the standard deviation of the respective signals:

$$
\begin{aligned}
\gamma\left(l, m, y_{p}\right)= & \frac{I\left(l, m, y_{p}\right)-\bar{I}\left(l, m, y_{p}\right)}{\Delta I\left(l, m, y_{p}\right)} \\
& \otimes \frac{I\left(l, m^{\prime}, y_{p}\right)-\bar{I}\left(l, m^{\prime}, y_{p}\right)}{\Delta I\left(l, m^{\prime}, y_{p}\right)},
\end{aligned}
$$

where $\bar{I}$ and $\Delta I$ are the mean and standard deviation of the signals, respectively, $\otimes$ is the cross-correlation operation, and the correlation coefficient $\gamma\left(l, m, y_{p}\right)$ ranges from -1 to +1 . The maximum value of the correlation coefficient $\left(M_{l, m}\right)$ is unity, and occurs when the corresponding coefficients $a_{n}$ are the same between $I\left(l, m, y_{p}\right)$ and $I\left(l, m^{\prime}, y_{p}\right)$. This implies that only a phase shift is involved between the two signals. Here, the phase shift $v(l, m)$ can be assumed to be the same for all orders, and the phase shift coefficient for the two signals can be written as

$$
\varphi_{n}\left(l, m^{\prime}\right)=\varphi_{n}(l, m)+v(l, m)
$$

$v(l, m)$ can then be used to calculate the local wave front radius of curvature, which is related to the wave front gradient $[11,19]$. The maximum value of the correlation coefficient is reduced if the difference of the coefficient $a_{n}$ between two nearby pixels increases due to strong distortion of the speckle pattern. The relative decrease of the correlation coefficient due to the specimen can be used to quantify the loss of the coherence, and the normalized maximum correlation coefficient $M_{l, m}$ can be defined as

$$
M_{l, m} \equiv M_{l, m}^{s} / M_{l, m}^{r},
$$

where $s$ and $r$ denote the sample and reference beams, respectively.

Dark-field image contrast increases when the maximum correlation coefficient $M$ reduces due to internal inhomogeneity in the specimen. As mentioned above, grating interferometry is treated as a special case of the specklebased technique. Hence, the normalized maximum correlation coefficient $M$ for the proposed technique is analogous to the fringe visibility $V$ for grating interferometry. The dark-field signal $D$ can be defined via the relation [20]

$$
D=-2 \log M .
$$

Although several physical phenomena can contribute to the reduction of the correlation coefficient, we consider only the two dominant phenomena: small-angle scattering and second-order differential phase contrast [21]. In particular, the internal electron density fluctuations in the specimen produce small-angle $\mathrm{x}$-ray scattering, which, in turn, distorts the speckle pattern. The second-order differential phase contrast from a specimen is often observed under illumination using a partially coherent $\mathrm{x}$-ray beam. The speckle pattern abruptly changes around the edges of the specimen, creating an interference pattern. Hence, the contribution from the second-order differential phase contrast is also included in the calculation of the correlation coefficients.

The principle of the technique presented was validated with experimental measurements at the Diamond Light Source's B16 Test beamline [22]. X-rays with an energy of $21 \mathrm{keV}$ were selected from the bending magnet source using a silicon double-crystal monochromator. A schematic of the experimental setup is shown in Fig. 1. The specimen was mounted on a motorized stage located $47 \mathrm{~m}$ from the x-ray source. A membrane was mounted on a twodimensional piezoelectric stage installed $100 \mathrm{~mm}$ downstream of the specimen. The distance between the specimen and detector was $1000 \mathrm{~mm}$. Images of the speckle pattern were collected using a high-resolution x-ray camera composed on a PCO 4000 CCD detector and a microscope objective with a Ce-doped YAG scintillator. Two stacks of images, with and without the specimen, were acquired by scanning the membrane transverse to the $\mathrm{x}$-ray beam for the horizontal and vertical dark-field images. As a 


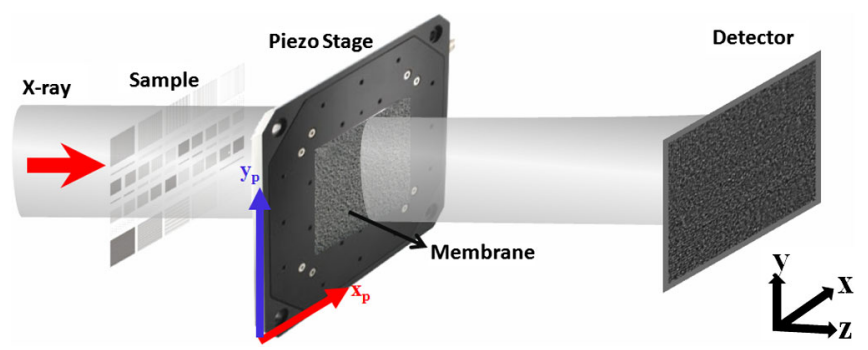

FIG. 1 (color online). A schematic of the experimental setup. The sample is placed upstream of a phase membrane, which is mounted on a precision piezoelectric motion stage. The $\mathrm{X}$ ray speckle pattern is recorded using a high-resolution area detector.

demonstration of the capabilities of the new technique, we first compared a rubber slice with a thin PTFE sheet. Samples were purposefully chosen, as they have considerably different small-angle scattering properties, even though absorption is similar. The Micro-CT BarPattern NANO Phantom (QRM, Germany) with transverse bar patterns was also studied, and the directional dark-field signal was demonstrated.

The PTFE and rubber samples were mounted side by side and exposed to $\mathrm{x}$ rays simultaneously. The rubber slice was cut so that the specimen thickness varied from the edge to the center. A $4 \times$ microscope objective provided an effective pixel resolution of $2.25 \times 2.25 \mu \mathrm{m}^{2}$. Sixty images for each stack were collected with a step size of $0.5 \mu \mathrm{m}$. Images were integrated over a $10 \mathrm{~s}$ acquisition period. The membrane, with an average pore size of $5 \mu \mathrm{m}$, was scanned along the transverse direction $y_{p}$ over a distance of $30 \mu \mathrm{m}$. The stack of speckle images is shown in Fig. 2(a). A speckle pattern is clearly visible in the zoomed region shown in Fig. 2(b). Here, we define the speckle visibility $V$ as the ratio $V=\left(I_{\max }-I_{\min }\right) /\left(I_{\max }+I_{\min }\right)$ where $I_{\max }$ and $I_{\min }$ correspond to the maximum and minimum intensities in the region of interest. It can be seen from the speckle intensity profile [Fig. 2(b), bottom] that speckle visibility in the PTFE sample is similar to empty space, whereas speckle visibility through the rubber is considerably less. Furthermore, the speckle pattern is significantly smeared on the right side of the rubber because of the drastic decrease of coherence due to the higher scattering from the thicker region of the rubber. Intensity oscillations in two nearby pixels for the PTFE and rubber samples are plotted as a function of $y_{p}$ in Fig. 2(c). It can be seen that the PTFE curves have similar shapes and are shifted relative to each other. This implies that small-angle scattering from the PTFE only marginally changes the speckle pattern. In contrast, the two curves for the rubber sample [Fig. 2(c), bottom] are very different. Correlation coefficient curves for the two specimens derived by applying the cross-correlation algorithm are shown in Fig. 2(d). The maximum value of the correlation coefficient for PTFE (0.97) is much higher than for rubber (0.41). The dark-field image was then extracted from this maximum
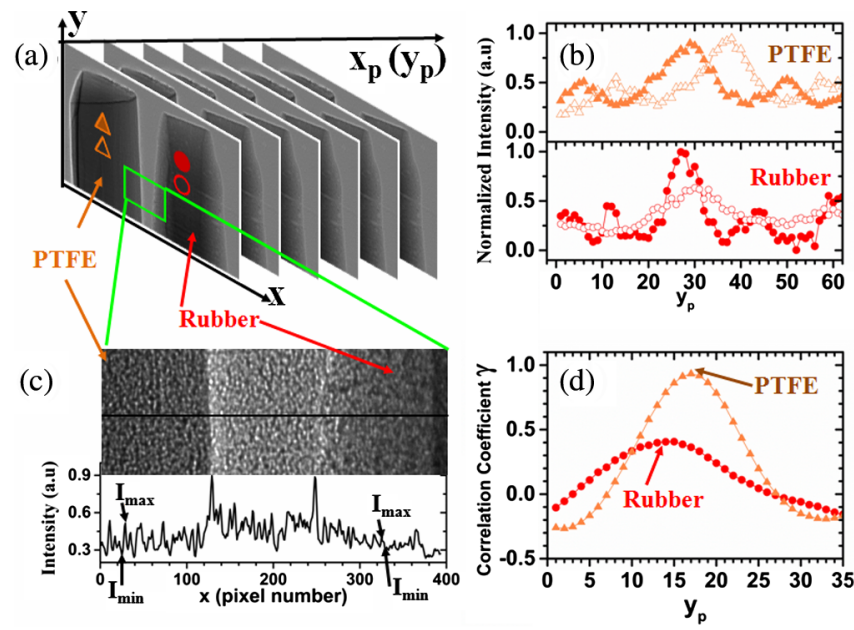

FIG. 2 (color online). (a) A stack of speckle images for a PTFE and a rubber sample (b) speckle image and intensity profile in the zoomed region. (c),(d) are the speckle intensity scans and corresponding correlation coefficient at the PTFE and the rubber locations.

correlation coefficient using Eq. (4) and performing pixelwise analysis.

Figure 3 shows the absorption and edge-enhancement images and vertical dark-field images for the two specimens. Figure 3(a) illustrates that although the two samples have different internal compositions, they have very similar absorption properties. However, Fig. 3(b) shows that the two samples have very different scattering properties. As expected, the homogeneous density distribution in PTFE does not create significant scattering. However, because the microstructure of rubber is porous and inhomogeneous, it produces significant small-angle scattering. Enhanced scattering in the dark-field image near to the top of PTFE is due to a rougher edge caused by cleaving the PFFE sheet by hand. Again, this fine detail is not visible in the absorption image. Hence, it is clear that the dark-field image generated by using the speckle pattern provides
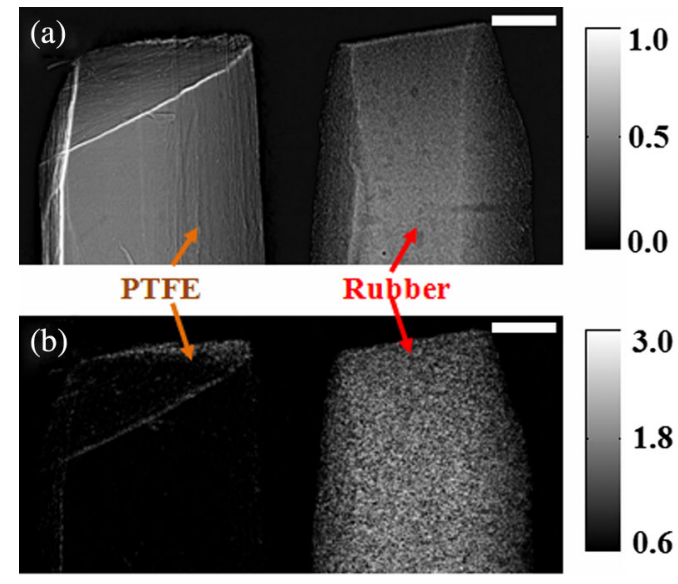

FIG. 3 (color online). (a) Absorption and (b) vertical dark-field image for PTFE and rubber samples. The scale bar is $0.5 \mathrm{~mm}$. 
(a)

\begin{tabular}{|c|c|}
\hline 0.4 & 0.5 \\
\hline$\mu \mathrm{m}$ & $\overline{85}$ \\
\hline
\end{tabular}

(c)

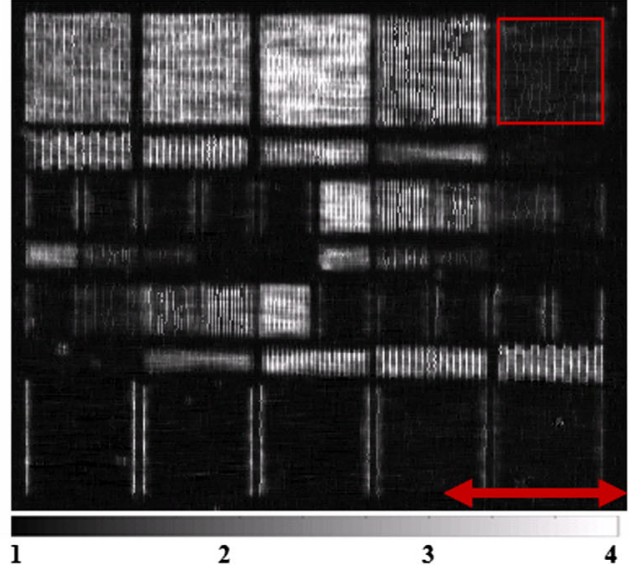

0.7

(b)

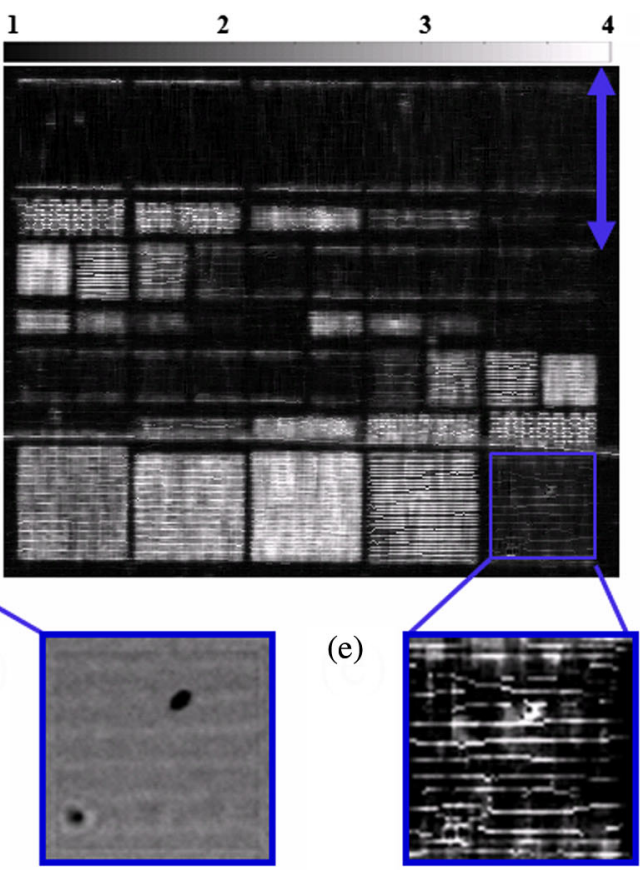

(f)

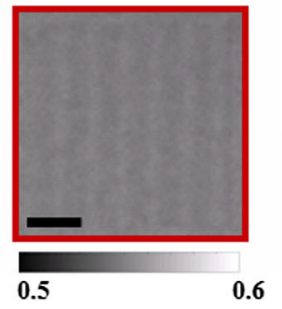

(g)

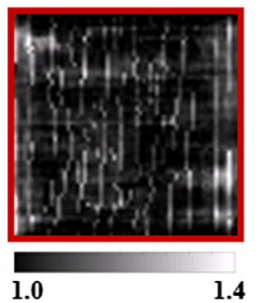

FIG. 4 (color online). (a) Absorption image, vertical (b), and horizontal (c) dark-field images of a bar-pattern phantom. Two-times magnified and contrast-optimized parts of the absorption image [(d),(f)] and directional dark-field image [(e),(g)]. (d),(e) and (f),(g) show the horizontal and vertical bar patterns with a $2 \mu \mathrm{m}$ line width, respectively. The scale bar is equal to $0.2 \mathrm{~mm}$.

significantly enhanced contrast compared to a conventional absorption image.

A bar-pattern phantom was selected to demonstrate the applicability of the dark-field technique for the study of strongly ordered systems. This phantom is composed of two $3 \times 3 \mathrm{~mm}^{2}$ chips, which contain several line and point patterns with features of 1 to $10 \mu \mathrm{m}$ wide. In order to show the capability to inspect the porosity at a subpixel length scale, a $10 \times$ microscope objective was chosen to provide an effective pixel resolution of $3.6 \times 3.6 \mu \mathrm{m}^{2}$ by $4 \times 4$ pixel binning. A sheet of silicon carbide abrasive paper with average particle diameter of $18.3 \mu \mathrm{m}$ was used so that the speckle pattern could be resolved by the detector. Exposure time for each image was $2 \mathrm{~s}$, and 100 images for each stack, with and without the sample, were acquired by scanning the membrane transverse to the x-ray beam. In the conventional absorption image, Fig. 4(a), lines and points with thickness greater than $4 \mu \mathrm{m}$ are visible with lower contrast. Figures 4(b) and 4(c) represent the vertical and horizontal dark-field images, respectively. As expected, the two directional dark-field images provide complementary information. In principle, a locally strongly oriented sample scatters primarily perpendicular to the axial direction creating the observed contrast [4]. In Fig. 4(b), vertical scattering is clearly visible for the horizontal lines, while they are hardly noticeable in the horizontal dark-field images [Fig. 4(c)]. In contrast, the vertical lines in Fig. 4(b) show much less contrast than in Fig. 4(c). Therefore, the speckle scanning technique provides directional dark-field images and gives insight into the orientation of the edges, fibers, or cracks in the specimen. By looking closely at the sections of the images [enclosed by the square regions in Figs. 4(a)-(c))], a dramatically different behavior in the absorption and scattering information for the two directions is observed. The lines in the marked square in Fig. 4(a) are not present in the absorption image [Figs. 4(d) and 4(f)] since the line width is only $2 \mu \mathrm{m}$, which is smaller than the detector pixel size. In contrast, the scattering signals of these thinner lines are clearly visible in the corresponding dark-field images [Figs. 4(e) and 4(g)].

In summary, we have demonstrated that the speckle scanning technique can be used for directional dark-field imaging with pixel size spatial resolution, which enables one to detect fine structures in materials. The technique requires 
only moderate $\mathrm{x}$-ray beam coherence and a simple experimental setup compared to the other dark-field imaging techniques. In addition, quantitative scattering information can potentially be derived from the dark-field signal. Moreover, the proposed approach of using a 1D scan is highly desirable for rapid and reduced-dose x-ray imaging since it is much quicker than the conventional standard deviation method employing a 2D raster scan. Furthermore, 1D scanning enables directional $x$-ray dark-field imaging of anisotropic samples. The technique can be employed for the nondestructive study of a material's porosity and aging properties. The technique further benefits from providing both the dark-field image and the differential phase-contrast image simultaneously from the same data set [11]. As the phase contrast and the dark-field images provide complementary information, a more detailed characterization of the sample is achieved (see the Supplemental Material [12]). The method is equally applicable for the inspection of biomedical and material science samples.

This work was carried out with the support of Diamond Light Source Ltd. The authors are grateful to Sebastien Berujon from ESRF for fruitful discussion and Simon Alcock for correcting the manuscript.

*hongchang.wang@diamond.ac.uk

[1] V. Revol, I. Jerjen, C. Kottler, P. Schütz, R. Kaufmann, T. Lüthi, U. Sennhauser, U. Straumann, and C. Urban, J. Appl. Phys. 110, 044912 (2011).

[2] E. Pagot, P. Cloetens, S. Fiedler, A. Bravin, P. Coan, J. Baruchel, J. Härtwig, and W. Thomlinson, Appl. Phys. Lett. 82, 3421 (2003).

[3] F. Pfeiffer, M. Bech, O. Bunk, P. Kraft, E. F. Eikenberry, C. Bronnimann, C. Grunzweig, and C. David, Nat. Mater. 7, 134 (2008).

[4] T. H. Jensen et al., Phys. Rev. B 82, 214103 (2010).

[5] F. Bayer, S. Zabler, C. Brendel, G. Pelzer, J. Rieger, A. Ritter, T. Weber, T. Michel, and G. Anton, Opt. Express 21, 19922 (2013).
[6] S. Schleede et al., Proc. Natl. Acad. Sci. U.S.A. 109, 17880 (2012).

[7] V. Revol, C. Kottler, R. Kaufmann, A. Neels, and A. Dommann, J. Appl. Phys. 112, 114903 (2012).

[8] K. Scherer et al., PLoS One 9, e93502 (2014).

[9] M. Endrizzi, P. C. Diemoz, T. P. Millard, J. Louise Jones, R. D. Speller, I. K. Robinson, and A. Olivo, Appl. Phys. Lett. 104, 024106 (2014).

[10] F. A. Vittoria, M. Endrizzi, P. C. Diemoz, U. H. Wagner, C. Rau, I. K. Robinson, and A. Olivo, Appl. Phys. Lett. 104, 134102 (2014).

[11] S. Berujon, H. Wang, and K. Sawhney, Phys. Rev. A 86, 063813 (2012).

[12] See Supplemental Material at http://link.aps.org/ supplemental/10.1103/PhysRevLett.114.103901 for comparison between the proposed technique and the previous method using standard deviation. An example is also given to demonstrate that dark-field and phase contrast images can provide complementary information over the absorption image.

[13] I. Zanette, T. Zhou, A. Burvall, U. Lundström, D. H. Larsson, M. Zdora, P. Thibault, F. Pfeiffer, and H. M. Hertz, Phys. Rev. Lett. 112, 253903 (2014).

[14] M. Giglio, M. Carpineti, and A. Vailati, Phys. Rev. Lett. 85, 1416 (2000).

[15] X. Lu, S. G. J. Mochrie, S. Narayanan, A. R. Sandy, and M. Sprung, J. Synchrotron Radiat. 18, 823 (2011).

[16] R. Cerbino, L. Peverini, M. A. C. Potenza, A. Robert, P. Bosecke, and M. Giglio, Nat. Phys. 4, 238 (2008).

[17] J. W. Goodman, Statistical Optics (John Wiley \& Sons, New York, 1985).

[18] T. Weitkamp, A. Diaz, C. David, F. Pfeiffer, M. Stampanoni, P. Cloetens, and E. Ziegler, Opt. Express 13, 6296 (2005).

[19] S. Berujon, H. Wang, S. Alcock, and K. Sawhney, Opt. Express 22, 6438 (2014).

[20] Z.-T. Wang, K.-J. Kang, Z.-F. Huang, and Z.-Q. Chen, Appl. Phys. Lett. 95, 094105 (2009).

[21] Y. Yang and X. Tang, Med. Phys. 39, 7237 (2012).

[22] K. J. S. Sawhney, I. P. Dolbnya, M. K. Tiwari, L. Alianelli, S. M. Scott, G. M. Preece, U. K. Pedersen, and R. D. Walton, AIP Conf. Proc. 1234, 387 (2010). 